\title{
Continuous sub-culture for investigation of stable strain of Streptomyces sp. KB1 for bioactive secondary metabolites Production
}

\author{
Monthon Lertcanawanichakul ${ }^{1,2 \star}$ and Kittisak Chawawisit ${ }^{1,2}$ \\ 'School of Allied Health Sciences, Walailak University, Nakhon Si Thammarat 80161, \\ Thailand \\ ${ }^{2}$ The Research Unit of Natural Products Utilization, Walailak University, Nakhon Si \\ Thammarat 80161, Thailand
}

\begin{abstract}
The bacterial isolate from air was identified as Streptomyces sp. KB1 based on the analysis of the $16 \mathrm{~S}$ rDNA sequence and collected as type strain TISTR2304 at Thailand Institute of Scientific and Technological Research (TISTR). It produced bioactive secondary metabolites that showed broad spectrum of antimicrobial activity. Stable strain of Streptomyces sp. KB1 was investigated for bioactive secondary metabolites production by continuous subculture method. Population of stable strain that showed parental levels of anti-Staphylococcus aureus TISTR 517 activity was continuously sub-cultured for 3-rounds and incubation time of each round as 5 days. Round I, II and Round III showed ratio of stable and non-stable strain as 1:2, 1:2 and 5:4, respectively. Moreover, stable strain of Round III, isolate no. 1-3-6-4, also showed activity higher than parental levels. These preliminary results might be led to solving the instability of bioactive secondary metabolites production of Streptomyces sp. in the future. Key words : Anti-Staphylococcus aureus activity; Bioactive secondary metabolites; Continuous Sub-Culture; Streptomyces sp. KB1.
\end{abstract}

\section{Introduction}

Streptomyces is Gram-positive, free-living, saprophytic bacteria, widely distributed in soil, water and colonizing plants ${ }^{1-3}$. Today, Streptomyces sp. is of considerable industrial importance because they produce important bioactive secondary metabolites such as antifungal, antiviral, antitumoral, anti-hypertensive, immunosuppressive agent and mainly antibiotics ${ }^{4}$. Bioactive secondary metabolites, also known as natural products, are produced by a cell through the process of cellular metabolism that is not essential for normal growth. They are not necessary to the cells survival itself but are more so for that of the entire microorganism or produced when the cell is not operating under optimum conditions such as nutrient source depletion. Therefore, the most of bioactive secondary metabolites have been produced during the end or near the stationary phase of growth for killing or inhibiting the growth of the related microorganism in inappropriate environment ${ }^{5}$. Currently, bioactive secondary metabolites have been studied worldwide. However, there was a report about wild-type strains of Streptomyces spp. which isolated from nature have not stability in bioactive secondary metabolites production when they were 
cultivated in unlimited medium and without competition ${ }^{6}$. Moreover, the instability of bioactive secondary metabolites production has been suggested to be associated with genetic instability. In the most of Streptomyces spp., they exhibit an extraordinarily high genetic instability rate in certain genes involved in their both primary metabolism and secondary metabolic pathways. Unstable genetic systems that have been initially studied in Streptomyces spp. were often attributed to plasmid loss. However, in many species, evidence has now accumulated of chromosomal DNA changes including deletions and amplifications. The characters that are subjected to instability mainly affect secondary metabolic functions such as pigment production or bioactive secondary metabolites production ${ }^{7}$. For example, the population of non-turimycin-producing strain of $S$. hygroscopicus, usually produced turimycin antibiotic, overgrew more than population of turimycin-producing strain when it was continuously cultured under time-unlimited condition ${ }^{6}$. Also, research of Gravius et al found that during a strain selection program to improve oxytetracycline production in Streptomyces rimosus R6, it showed extreme morphological instability. They reported that Round I of cultivation of S. rimosus R6 (99\% of variants) showed parental levels of resistance but were very heterogeneous regarding the other phenotypes, Round II of cultivation of S. rimosus R6 (1\% of variants) showed phenotypically uniform, and most variants carried the same large depletion and Round III of cultivation of $S$. rimosus R6 ( $0.1 \%$ of variants) showed phenotypically uniform and overproduced oxytetracycline antibiotic ${ }^{8}$. While, the variance of Streptomyces sp. KB1 related with bioactive secondary metabolites production. Streptomyces sp. KB1 could produce the important bioactive secondary metabolites that showed broad spectrum of antimicrobial activity against representative of Grampositive bacteria (S. aureus TISTR 517, Micrococcus luteus TISTR 884 and Bacillus cereus TISTR 11778), Gram-negative bacteria (Escherichia coli TISTR 887, Salmonella typhimurium TISTR 292 and Pseudomonas aeruginosa TISTR 1467) and yeasts (clinical isolate of Candida albicans and Cryptococcus neoformans). Unfortunately, it has not the stability of production. Therefore, the study was aimed for investigation of stable strain of Streptomyces sp. KB1 that has the stability of bioactive secondary metabolites production by means of continuously sub-culturing method under time-limited condition.

\section{Material and Methods}

\section{Microorganisms, Media and Cultural conditions}

Streptomyces sp. KB1 was kindly gifted from Associate Professor Dr. Monthon Lertcanawanichakul, School of Allied Health Sciences, Walailak University, Thailand. The isolate KB1 was identified by partial 16S rDNA gene sequence analysis at National Center for Genetic Engineering and Biotechnology (BIOTEC), Pathum Thani province, Thailand, and submitted to GenBank database (NCBI, USA), accession number KF939581.1. It was cultured in half-formula of Luria Bertani (LB/2; Himedia, India) agar medium (5 g/l Tryptone, $2.5 \mathrm{~g} / 1$ Yeast Extract, $5 \mathrm{~g} / \mathrm{l} \mathrm{NaCl}$ and $15 \mathrm{~g} / \mathrm{l}$ Agar powder) at $30{ }^{\circ} \mathrm{C}$ in static incubator (WTB binder, Germany) for 5 days. After incubation, single colony was inoculated into $10 \mathrm{ml}$ of LB/2 broth medium in $25 \times 150 \mathrm{~mm}$ of screw cap test tube, incubated at $30{ }^{\circ} \mathrm{C}, 200$ revolutions per minute (RPM) in shaking incubator (481, Thermo scientific, USA) for 2 days or until the cells grown up to late logarithmic phase and stored in $15 \%$ glycerol at $-80{ }^{\circ} \mathrm{C}$ until use.

Staphylococcus aureus TISTR 517 which was assigned as indicator bacteria was cultured in full-formula of Luria Bertani (LB) agar medium at $37^{\circ} \mathrm{C}$ in static incubator for 24 hours. After incubation, single colony was inoculated into $10 \mathrm{ml}$ of LB broth medium in $25 \times 150 \mathrm{~mm}$ of screw cap test tube, incubated at $37{ }^{\circ} \mathrm{C}, 200 \mathrm{RPM}$ in shaking incubator for 24 hours or until the cells grown up to late logarithmic phase and then stored in $15 \%$ glycerol at $-80{ }^{\circ} \mathrm{C}$ until use.

\section{Phylogenetic Analysis}

Almost complete sequence (1,445 bp) of 16S rDNA gene was determined and compared with those of other closely related taxa retrieved from the GenBank database. Phylogenetic tree was constructed by Neighbour-Joining plot. A sequence similarity search was done using GenBank BLASTN (www.ncbi.nlm.nih.gov/blst). Sequences of closely related taxa were retrieved and aligned by means of MEGA 4 program. For the Neighbour-joining analysis, the distances between the sequences were calculated using Kimura's two-parameter model. Bootstrap analysis was performed to assess the confidence limits of the branching. 


\section{Investigation of stable strain}

i) Starter, Round I, II and Round III preparation

a.) Starter preparation: Briefly, $1 \times 1 \mathrm{~cm}^{2}$ of initial streak of 5-days culture of Streptomyces sp. KB1 was inoculated to $10 \mathrm{ml}$ of LB/2 broth medium in $25 \times 150 \mathrm{~mm}$ of screw cap test tube. Inoculum was incubated at 30 ${ }^{\circ} \mathrm{C}, 200$ RPM in shaking incubator for 5 days and assigned as starter or Round 0.

b.) Round I preparation: Briefly, $1 \%$ of starter was sub-cultured to $10 \mathrm{ml}$ of LB/2 broth medium in $25 \times 150 \mathrm{~mm}$ of screw cap test tube (6-tubes) and then incubated at $30{ }^{\circ} \mathrm{C}, 200 \mathrm{RPM}$ in shaking incubator for 5 days. After incubation, $200 \mu \mathrm{l}$ of culture broth of each tube was separated the cell sediment by using centrifugation at 12,000 RPM for 20 minutes and then tested the anti- S. aureus TISTR 517 activity by using agar well diffusion method. Tube that showed activity was collected to prepare Round II.

c.) Round II and III preparation: Preparation of Round II and III sub-culturing of Streptomyces sp. KB1 is the same as sub-culturing Round I from Round 0 . The flow chart of preparation of Round I, II, and Round III subculturing of Streptomyces sp. KB1 was illustrated in Figure 1.

ii) Anti-S. aureus TISTR 517 activity test

Anti- S. aureus TISTR 517 activity was determined by means of agar well diffusion method as described by Kekuda et al. ${ }^{9}$. Briefly, single colony of 24 hours-culture of $S$. aureus TISTR 517 was inoculated to $5 \mathrm{ml}$ of LB broth medium in $15 \times 150 \mathrm{~mm}$ of screw cap test tube and then incubated at $37{ }^{\circ} \mathrm{C}, 200 \mathrm{RPM}$ in shaking incubator for 24 hours. After incubation, cell suspension was approximately adjusted to a 0.5 McFarland standard turbidity by $0.85 \% \mathrm{NaCl}$ and swabbed on sterilized Mueller Hinton agar (MHA: Himedia, India) plates using sterile cotton swab and drilled well by a $5 \mathrm{~mm}$ in diameter of cork-borer. Fifty microliter of culture broth of each round from i) were added into the well. The plates were incubated at $37{ }^{\circ} \mathrm{C}$ in static incubator. After 24 hours of incubation, the plates were examined the zone of clearance around the each wells.

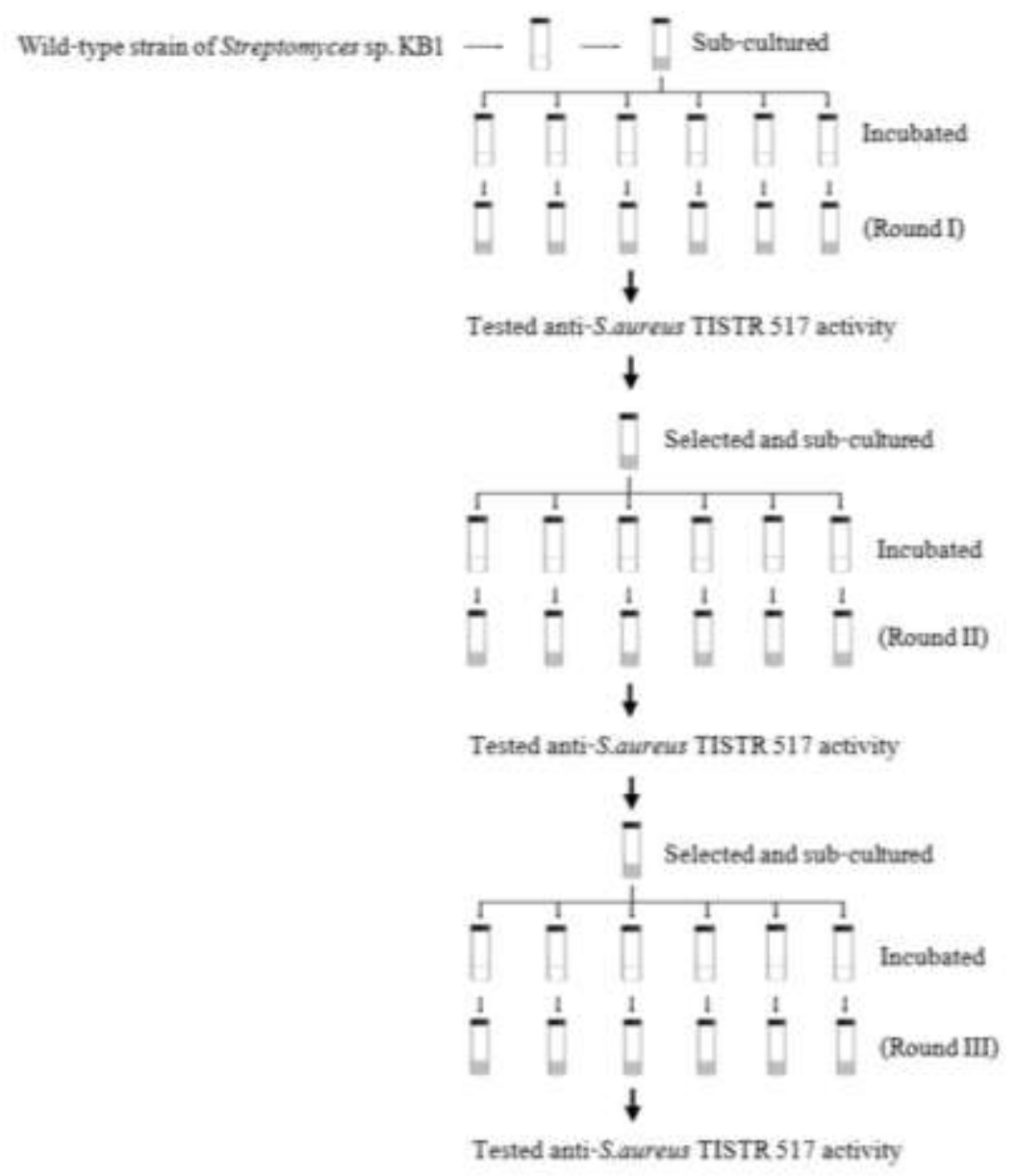

Figure 1 Scheme of investigation of stability producing-strain of Streptomyces sp. KB1 


\section{Results and Discussion}

From preliminary investigation it was found that the isolate KB1 is one of an important bioactive secondary metabolites-producing strain because it showed broad spectrum of antimicrobial activity against representative of Gram-positive bacteria, Gram-negative bacteria and yeasts (data not show). After 16S rDNA gene amplification, the resulted sequence was subjected to similarity searches against public databases to infer possible phylogenetic relationship of the isolate KB1. The phylogenetic tree (Figure 2) from representative strains of the related species indicated that the isolate KB1 should be placed in the genus Streptomyces. In the comparison of $16 \mathrm{~S}$ rDNA gene sequences, the isolate KB1 was mostly related with $S$. varsoviensis NRRL B$3589^{\mathrm{T}}(98.89 \%)$, S. youssoufiensis $\mathrm{X} 4^{\mathrm{T}}(98.68 \%)$ and S. ramulosus NRRL B- $2714^{\mathrm{T}}(98.61 \%)$. While, Kitasatospora setae KM- $6054^{\mathrm{T}}$ was used as an out-group. Based on the morphology and molecular phylogeny, the isolate KB1 was identified as genus Streptomyces and named as Streptomyces sp. KB1. The 16S rDNA gene sequence of Streptomyces sp. KB1 has been deposited in the GenBank database under the accession number KF939581.1. The culture of KB1 was deposited at TISTR as type strain TISTR2304.

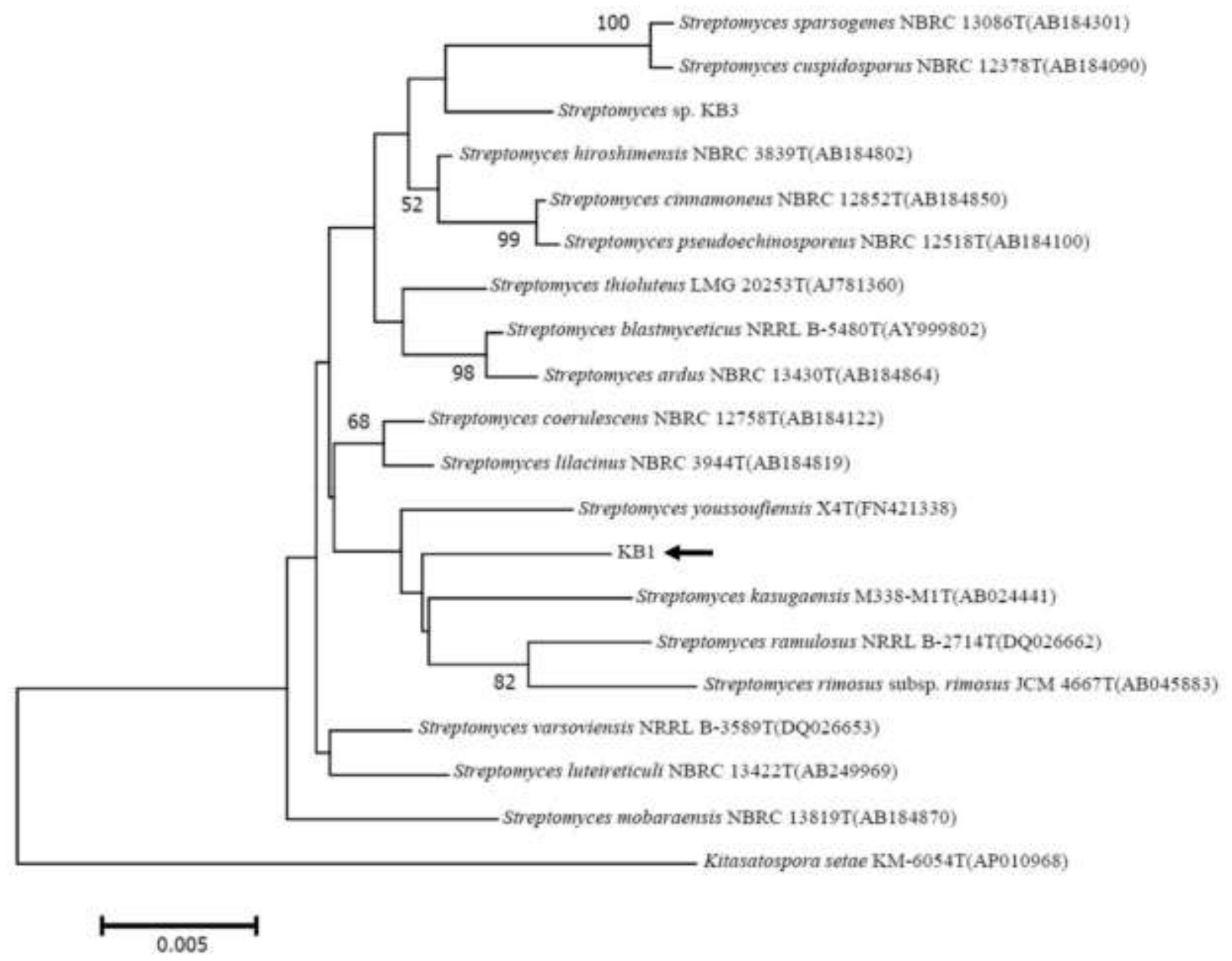

Figure 2: The phylogram showing the position of the isolate KB1 with other Streptomyces based on $16 \mathrm{~S}$ rDNA gene. Phylogenetic tree based on neighbor-joining analysis of 1000 resampled data. Number at nodes indicates the percent level of bootstrap support. The scale bar represents 0.005 substitutions per nucleotide position.

Wild-type strain of Streptomyces sp. KB1 (starter or Round 0) was investigated for the strain instability by sub-culturing for 3-rounds (Round I, II and Round III). It indicated that Round I, II and Round III of sub-culturing of Streptomyces sp. KB1 likened that they came from the same origin (starter or Round 0). Whereas, after subcultured the cultures from starter into the freshly prepared LB/2 broth medium for continuing growth to Round II and Round III. It was found that the producing strain of Streptomyces sp. KB1 was not stability for bioactive secondary metabolites. It implied that Streptomyces sp. KB1 had two populations including bioactive secondary 
metabolites-producing population and bioactive secondary metabolites non-producing population. Both two populations were morphologically confirmed by streak plate method found that they are alike (data not show). These results accorded with research of Roth et al. that reported the population of non-turimycin-producing strain of S. hygroscopicus, usually produced turimycin antibiotic, overgrew more than turimycin-producing strain when it was continuously cultured under time-unlimited condition ${ }^{6}$.

Table 1: Anti- S. aureus TISTR 517 activity of Streptomyces sp. KB1 variants

\begin{tabular}{|c|c|c|}
\hline $\begin{array}{c}\text { Round of sub-culturing of } \\
\text { producing-strain }\end{array}$ & $\begin{array}{c}\text { Isolate } \\
\text { designation }\end{array}$ & $\begin{array}{c}\text { Anti- } \begin{array}{c}\text { S. aureus TISTR } 517 \\
\text { activity }\end{array} \\
\end{array}$ \\
\hline Starter (Round 0) & 1 & +++ \\
\hline \multirow[t]{6}{*}{ Round I } & $1-1$ & - \\
\hline & $1-2$ & - \\
\hline & $1-3 *$ & +++ \\
\hline & $1-4$ & - \\
\hline & $1-5^{*}$ & +++ \\
\hline & $1-6$ & - \\
\hline \multirow[t]{6}{*}{ Round II } & $1-3-1 *$ & - \\
\hline & $1-3-2$ & - \\
\hline & $1-3-3 *$ & +++ \\
\hline & $1-3-4$ & - \\
\hline & $1-3-5$ & - \\
\hline & $1-3-6^{*}$ & +++ \\
\hline \multirow[t]{6}{*}{ Round II } & $1-5-1$ & - \\
\hline & $1-5-2 *$ & +++ \\
\hline & $1-5-3$ & - \\
\hline & $1-5-4$ & - \\
\hline & $1-5-5$ & + \\
\hline & $1-5-6$ & - \\
\hline \multirow[t]{6}{*}{ Round III } & $1-3-3-1$ & - \\
\hline & $1-3-3-2$ & ++ \\
\hline & $1-3-3-3$ & - \\
\hline & $1-3-3-4$ & - \\
\hline & $1-3-3-5$ & ++ \\
\hline & $1-3-3-6$ & - \\
\hline \multirow{6}{*}{ Round III } & $1-3-6-1$ & ++ \\
\hline & $1-3-6-2$ & ++ \\
\hline & $1-3-6-3$ & ++ \\
\hline & $1-3-6-4$ & ++++ \\
\hline & $1-3-6-5$ & + \\
\hline & $1-3-6-6$ & + \\
\hline \multirow[t]{6}{*}{ Round III } & $1-5-2-1$ & - \\
\hline & $1-5-2-2$ & ++ \\
\hline & $1-5-2-3$ & - \\
\hline & $1-5-2-4$ & - \\
\hline & $1-5-2-5$ & + \\
\hline & $1-5-2-6$ & - \\
\hline \multirow{6}{*}{$\begin{array}{l}\text { Round III of sub-culturing of non } \\
\text { producing-strain }\end{array}$} & $1-3-1-1$ & - \\
\hline & $1-3-1-2$ & ++ \\
\hline & $1-3-1-3$ & + \\
\hline & $1-3-1-4$ & + \\
\hline & $1-3-1-5$ & - \\
\hline & $1-3-1-6$ & - \\
\hline
\end{tabular}

- No activity, +diameter of inhibition zone $<15 \mathrm{~mm},++$ diameter of inhibition zone in the range $15-20 \mathrm{~mm}$, +++ diameter of inhibition zone in the range $21-25 \mathrm{~mm},++++$ diameter of inhibition zone $>25 \mathrm{~mm}$

* Isolate was selected for continuously sub-cultured into next Round 
The instability of bioactive secondary metabolites production was suggested that it has been associated with genetic instability ${ }^{10}$. Genetic instability is very common in Streptomyces sp. and can affect a variety of gene. It has been affected different phenotypically properties, often pleiotropically, including morphological differentiation and production of bioactive secondary metabolites ${ }^{7-8,10}$. Genetic instability of most Streptomyces sp. came from three factors i) spontaneous mutation which had rate higher than $0.1 \%$ per spore ii) chemical- and physical- mutagenesis mutation and iii) $-20{ }^{\circ} \mathrm{C}$ storage mutation which were considered to associate between plasmid loss and chromosomal DNA depletion ${ }^{7}$. In nearly every well-characterized case of genetic instability in Streptomyces, the loss of gene function is due to depletion of the gene. In the case of S. glaucescens it is clear that the genetic instability involved with chromosomal DNA depletion. But, the location of the unstable genes is not clear. In addition, there is no physical evidence that the genes are plasmid encoded despite experiments to try to detect plasmid involvement ${ }^{6,8}$. Therefore, the instability of bioactive secondary metabolites production of Streptomyces sp. KB1 was assumed that might be related with depletion of bioactive secondary metabolites gene on chromosome. These assumptions were supported by the work from Aigle et al. which explained that the 8-9 $\mathrm{Mb}$ Streptomyces chromosome is linear, with a 'core' containing essential genes (including bioactive secondary metabolites gene) and 'arms' carrying conditionally adaptive genes ${ }^{11}$. Furthermore, Roth et al reported that occurrence of population of non-turimycin-producing strain during long continuous culture and they could not revert to turimycin-producing strain. This occurring was suggested that they had arisen by loss of genetic material on plasmid 6 . Contrast to this research, non-bioactive secondary metabolites-producing strain from Round II of sub-culturing (the designate isolate 1-3-1) was sub-cultured into the freshly prepared LB/2 broth medium. It was found that could be reproduced bioactive secondary metabolites (Table 1). From these result indicated that (i) bioactive secondary metabolites gene was reversible loss or (ii) these bioactive secondary metabolites were produced by function of silent gene that was stimulated. Because of Streptomyces species has showed that single specie could carry more than 30 unexpressed or silent gene clusters for bioactive secondary metabolite biosynthesis ${ }^{11}$. Unexpressed or silent gene was stimulated by physical (such as UV radiation) and chemical (such as Ethidium Bromide) stimulation ${ }^{7}$. So, expression of silent gene is one of three factors of genetic instability. Whereas, stimulated agent was not used in our research. Therefore, the reversion from Round II (non-bioactive secondary metabolites-producing strain) to Round III (bioactive secondary metabolites-producing strain) might be came from reversible loss of bioactive secondary metabolites gene which accorded with research of Gravius et al that reported revertants of some Round III variant showed a morphology and oxytetracycline production same to Round I variant and the aforementioned variance related with genetic instability in the chromosome ${ }^{8}$.

\section{Conclusion}

Streptomyces sp. KB1 is important bioactive secondary metabolites producer but it has both producing and non-producing strain. The stability strain of KB1 has been investigated by means of continuously subculturing method under time-limited condition.

\section{Acknowledgement}

The research was financial supported from Institute of Research and Development of Walailak University under the grant no. WU 60109. The author wish to thank the laboratory officer of faculty of Medical Technology, School of Allied Health Sciences, Walailak University, Nakhon Si Thammarat, Thailand, for providing facilities during this study.

\section{Conflict of interest}

The author(s) have no declared any conflict of interests.

\section{References}

1. D.A. Reardon, Oncologist, 11, 152 (2006); doi:10.1634/theoncologist.11-2-152.

2. H. Ohgaki and P. Kleihues, Clin. Cancer Res., 19, 764 (2013); doi:10.1158/1078-0432.CCR-12-3002.

3. Q.T. Ostrom, H. Gittleman, P. Farah, A. Ondracek, Y. Chen, Y. Wolinsky, N.E. Stroup, C. Kruchko and J.S. Barnholtz-Sloan, Neuro-oncol., 15, 1 (2013); doi:10.1093/neuonc/not151. 
4. C.B. Colen and E. Allcut, Neurosurg. Clin. N. Am., 23, 507 (2012); doi:10.1016/j.nec.2012.04.010.

5. V.T. Puliyappadamba, K.J. Hatanpaa, S. Chakraborty and A.A. Habib, Mol. Cell. Oncol., 1, e963478 (2014); doi:10.4161/23723548.2014.963478.

6. G.P. Atkinson, S.E. Nozell and E.T.N. Benveniste, Expert Rev. Neurother., 10, 575 (2010); doi:10.1586/ern.10.21.

7. F.B. Furnari, T. Fenton, R.M. Bachoo, A. Mukasa, J.M. Stommel, A. Stegh, W.C. Hahn, K.L. Ligon, D.N. Louis, C. Brennan, L. Chin, R.A. DePinho and W.K. Cavenee, Genes Dev., 21, 2683 (2007); doi:10.1101/gad.1596707.

8. S.J. Hentschel and F.F. Lang, Cancer J., 9, 113 (2003); doi:10.1097/00130404-200303000-00007.

9. Y.H. Kim and C.Y. Kim, Neurosurg. Clin. N. Am., 23, 199 (2012); doi:10.1016/j.nec.2012.01.010.

10. B.B. Taw, A.A. Gorgulho, M.T. Selch and A.A. De Salles, Neurosurg. Clin. N. Am., 23, 259 (2012); doi:10.1016/j.nec.2012.01.003.

11. S. Hayashi, M. Yamamoto, Y. Ueno, K. Ikeda, K. Ohshima, G.-I. Soma and T. Fukushima, Neurol. Med. Chir. (Tokyo), 41, 187 (2001); doi:10.2176/nmc.41.187.

12. P. Korkolopoulou, G. Levidou, A.A. Saetta, E. El-Habr, C. Eftichiadis, P. Demenagas, I. Thymara, K. Xiromeritis, E. Boviatsis, E. Thomas-Tsagli, I. Panayotidis and E. Patsouris, Hum. Pathol., 39, 1143 (2008); doi:10.1016/j.humpath.2008.01.020.

13. C.B. Colen and E. Allcut, Neurosurg. Clin. N. Am., 23, 507 (2012); doi:10.1016/j.nec.2012.04.010.

14. D.T. Nagasawa, F. Chow, A. Yew, W. Kim, N. Cremer and I. Yang, Neurosurg. Clin. N. Am., 23, 307 (2012); doi:10.1016/j.nec.2012.01.007.

15. R.G. Verhaak, K.A. Hoadley, E. Purdom, V. Wang, Y. Qi, M.D. Wilkerson, C.R. Miller, L. Ding, T. Golub, J.P. Mesirov, G. Alexe, M. Lawrence, M. O’Kelly, P. Tamayo, B.A. Weir, S. Gabriel, W. Winckler, S. Gupta, L. Jakkula, H.S. Feiler, J.G. Hodgson, C.D. James, J.N. Sarkaria, C. Brennan, A. Kahn, P.T. Spellman, R.K. Wilson, T.P. Speed, J.W. Gray, M. Meyerson, G. Getz, C.M. Perou and D.N. Hayes, Cancer Cell, 17, 98 (2010); doi:10.1016/j.ccr.2009.12.020.

16. A. Omuro, JAMA, 310, 1842 (2013); doi:10.1001/jama.2013.280319.

17. S. Ishkanian, N.J. Laperriere, W. Xu, B.-A. Millar, D. Payne, W. Mason and A. Sahgal, Cancer, 117, 4070 (2011); doi:10.1002/cncr.25988. 